\title{
Grammatical Gender Marking: The Gender Roles Mirror
}

\author{
Amir Shoham, Temple University, USA
}

The goal of this short article is to emphasize the ways in which the quantitative dimensions of language could help multinationals and foreigners in general to understand gender roles at the individual and society levels. Having an easy tool that captures gender roles but is not affected by current socio-economic cycles is crucial for preventing mistakes when conducting international activity. In this way, grammatical gender marking is important because of how the most significant aspects of culture in any society are gender roles. Without understanding the expected gender roles inherent in their counterparts and/or host societies, foreigners can make mistakes that can be costly at the individual and/or organization level. The roles of women and men in society have affected every civilization without exceptions via the cultural values and practices attributed to their gender. All of the major seminal studies of survey-based cultural dimensions include a dimension of gender (e.g., Hofstede, 1980) to highlight the importance of being aware of gender roles during business transactions. Moreover, because gender roles are so controversial and important in most societies, taking the wrong approach (from the viewpoint of the host society) may increase tension and conflict that could negatively impact the organization' goals and outcomes.

In recent years, a new quantitative tool emerged to capture gender roles in society based on grammatical gender markings in the linguistic structures of a language. The quantified dimensions of culture are important for academic research in the IB field, but nonetheless it is important for practitioners to have an objective and easy tool to rely on as the base for understanding gender roles in host societies. In particular, language has a significant impact on markets and organizations, and the grammatical gender marking method of evaluating gender roles in society quantifies gender structures in such a way that it can be used by academics in empirical research but can still be used by practitioners to identify the gender roles effecting foreigner counterparts and host societies.

Grammatical gender markings have a few major advantages over the traditional survey-based dimensions of culture. First, survey-based instruments are susceptible to severe endogeneity problems through the impact of current socio-economic conditions. Conversely, linguists largely agree that the grammatical gender structures of a language are among the most stable features of a language's grammar, one that is inherited from the distant past and capable of surviving for thousands of years (Wichmann \& Holman, 2009). This makes these grammars exogenous to changes in current socio-economic forces like business cycles or national news covering specific gender-related topics like rape or abortion rights.

Second, using grammatical structure could be conducted at the individual level without assuming a one-dimension score of culture for every individual in a country. For example, Hofstede (1980) has one Masculinity dimension for Nigeria, but Nigeria is a heterogeneous country with regard to gender roles. By using grammatical structures, we can capture differences in gender roles among ethnic groups, such as the Hausa and the Yoruba. Hicks, Santacreu-Vasut, and Shoham (2015) demonstrate that linguistic gender marking could capture gender roles at the individual level if the language that the individual speaks at home-or more importantly, the native tongue of the individual —is identified. This helps to avoid the homogeneity assumption for all the individuals in the nation/society that the survey-based dimensions of culture involve. Managers in multinational organizations seek accurate and easy ways to use tools to capture the cultural mindsets of employees from different cultural backgrounds. The survey-based dimensions are easy to use, but averaging at the national level reduces the tool's 
accuracy. The grammatical gender marking index that captures gender roles is easy to use and tailored to the regional/individual level.

Third, the World Atlas of Linguistic Structures (WALS) contains information on the structures of hundreds of languages, allowing researchers and practitioners to have much more detailed observations with a higher diversity than the survey-based cultural dimensions, which are available for far fewer societies and nations (e.g., GLOBE has 62 societies). This relates to the accuracy advantage of grammatical gender marking in that it avoids averaging the survey-based dimensions at the national/ country level.

Finally, Santacreu-Vasut, Shenkar, and Shoham (2014) and Shoham and Lee (2018) provide preliminary empirical results showing that the grammatical gender marking method is a better predictor of gender roles in society than the traditional survey-based dimensions offered by Hoftstede (MAS) and GLOBE (Gender egalitarianism). This result is not surprising given that grammatical gender marking probably captures ancestors' culture reinforced by cognition; in other words, it captures core values that are not affected by the current socio-economic conditions.
The following short description of grammatical gender marking explains the fundamental points of the method. (A more detailed explanation can be found in Santacreu-Vasut et al., 2014, and Gay, Santacreu-Vasut, \& Shoham, 2013.) WALS displays four grammatical structures related to gender. The first structure is Sex-Based (SB) gender (Corbett, 2011). A language's gender system can be based on biological sex or some other factor, like the distinction between humans and non-humans, or the distinction between animate and inanimate objects. For instance, the Danish and Swedish languages make distinctions that are not sex-based, but gender in English is based on biological sex. SB is a dummy variable that equals one for languages with a gender system based on biological sex, and zero for languages that are not.

The second structure is the number of genders (NG; Corbett, 2011). The number of genders is based on the quantity of noun-types that have different agreements; for example, French has two genders - "feminine/masculine" - whereas English includes a "neuter" gender. Some languages have many more, such as Nigerian Fula, which has 20 genders. NG is a dummy variable that equals one for languages with two genders, and zero for languages with fewer or more genders.

Figure 1. Grammatical Gender Marking

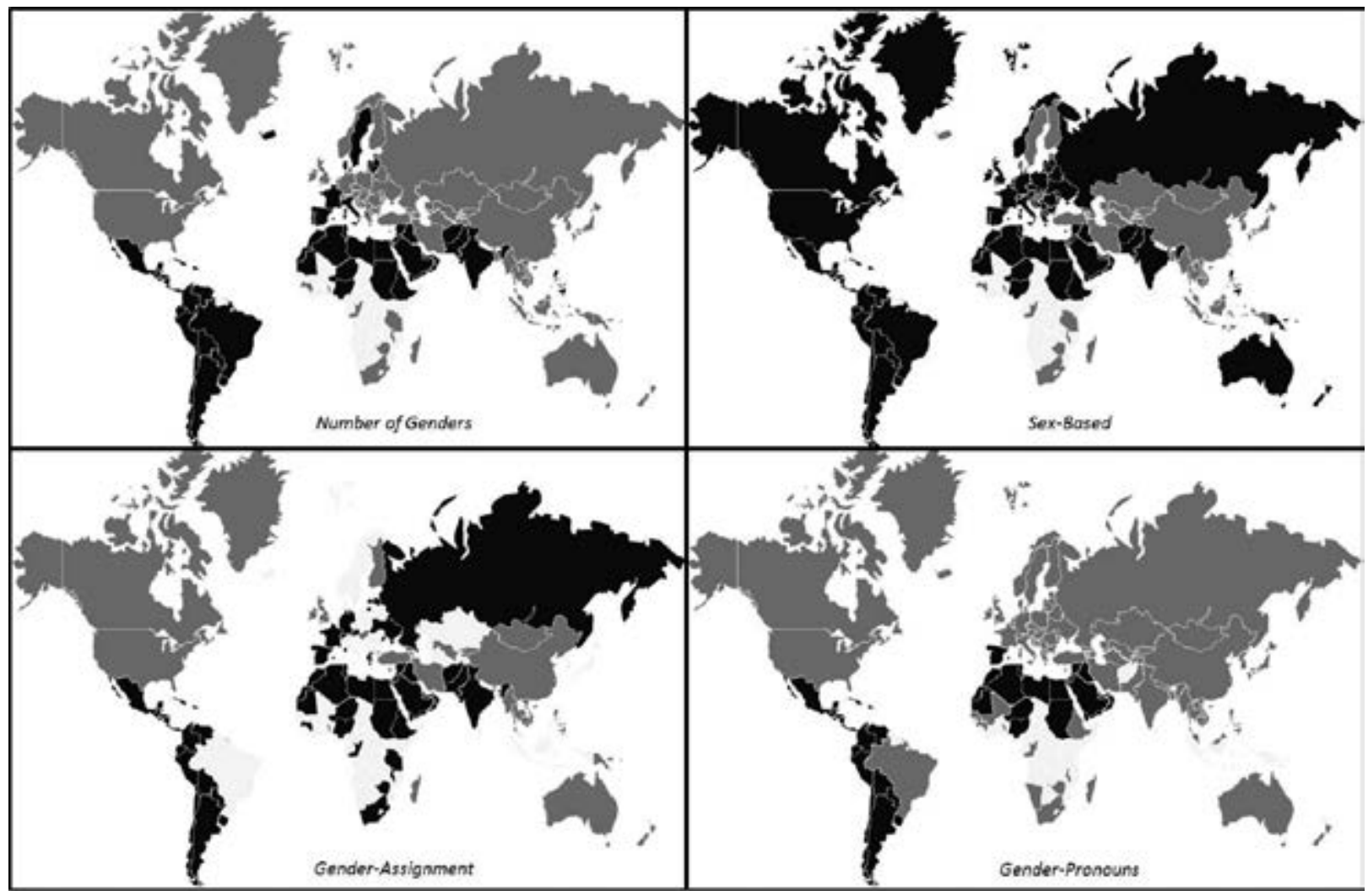

Note: The four gender structure Intensity Black countries means Dummy equals 1 (higher gender marking in the grammatical structure).

Source: Gay et al. (2013) 
The third structure is Gender Assignment (GA; Corbett, 2011), which captures how a speaker assigns nouns to the genders defined by the gender system of that language. A gender assignment system provides a set of rules to help the speaker make appropriate agreements. Assignment can depend on the meaning (semantic) or the form of the noun. For example, a "table" is neuter in English since the language assigns gender only on semantic grounds. However, it is feminine in French, which assigns gender to even nouns that do not have a biological gender. GA is a dummy variable that equals one for languages whose gender assignment system is both semantic and formal, and zero otherwise.

The fourth structure is Gender Pronouns (GP; Siewierska, 2011), which captures gender distinctions in independent personal pronouns. Some languages have no gender distinction in pronouns; other languages have gender distinctions only in third-person pronouns; and still others have gender distinctions in the third person and in the first and/or the second person. For example, English distinguishes gender only in third-person pronouns ("she," "he," and "it"). GP is a dummy variable that equals one for languages with gender distinction in the third, first, and/or second person pronouns, and zero otherwise.

From the four grammatical structures presented above, a gender intensity index (GII) was created, which is calculated thus: $\mathrm{GII}=\mathrm{NG}+\mathrm{SB}+\mathrm{GA}+\mathrm{GP}$, where GII can be any number between 0 and 4 (GII $\in\{0 ; 1 ; 2 ; 3 ; 4\})$. A higher score indicates greater emphasis placed on gender roles in a society. A score of 0 means no gender marking in the linguistic structures. A score of 4 means that all four structures in the language that are related to gender are based on biological sex. The maps in Figure 1 show the dummy variable for each of the four gender structures for each country's dominant language.

Table 1. Example of GII Scores by Language

\begin{tabular}{lccccc}
\hline Language & $\begin{array}{c}\text { GII } \\
\text { score }\end{array}$ & NG & SB & GA & GP \\
\hline Arabic & 4 & 1 & 1 & 1 & 1 \\
Spanish & 4 & 1 & 1 & 1 & 1 \\
Armenian & 0 & 0 & 0 & 0 & 0 \\
English & 1 & 0 & 1 & 0 & 0 \\
German & 2 & 0 & 1 & 1 & 0 \\
France & 3 & 1 & 1 & 1 & 0 \\
Mandarin & 0 & 0 & 0 & 0 & 0 \\
Greek & 2 & 0 & 1 & 1 & 0 \\
Finnish & 0 & 0 & 0 & 0 & 0 \\
Hebrew & 4 & 1 & 1 & 1 & 1 \\
\hline
\end{tabular}

Table 1 shows the GII for a few selected languages. The GII per country based on the dominant language could be obtained from Appendix B in Drori, Manos, Santacreu-Vasut, and Shoham (2019). For example, German has a GII of 2. German has a sex-based gender system $(\mathrm{SB}=1)$ and assigns gender on the basis of both semantic and formal rules $(\mathrm{GA}=1)$; however, German assigns gender to third-person pronouns only (GP = $0)$ and does have a neuter gender $(\mathrm{NG}=0)$.

As can be seen in Table 1, languages like Arabic, Spanish, and Hebrew have the highest grammatical gender marking replicating higher gender role societies than societies using Finnish as the dominant language. Corbett (2011) argues that gender "is the most puzzling of the grammatical categories," adding that whereas gender is evident in most phases in some languages, it is absent from other languages. Grammatical gender distinctions in languages arose from evolutionary pressures concerning specialization, reproduction, and the division of labor, which suggests that the use of gender in language may act as a cultural marker for historical gender norms. The persistent impact of ancestors' cultural values, marked in the grammar of a language, are reinforced by the impact of language on cognition. As grammar influences speakers' cognitive framework, it forces them to encode particular characteristics, and therefore shapes their mental representation of socio-economic reality.

As claimed above, a growing body of literature across different fields over the last decade uses grammatical gender marking to capture gender roles in a society. In the following paragraphs, I will present an overview of three papers that have already used grammatical gender marking and found interesting applied results that can be used by practitioners, policy makers or by instructors of IB looking for a different angle from which to approach cultural differences.

First, Santacreu-Vasut et al. (2014) show that grammatical gender marking of the dominant home country language of an MNC's HQ impacts gender staffing of subsidiary boards. Also, societies with higher linguistic gender marking in the dominant language have significantly lower female participation on boards of directors, executive suite, and smaller female-led corporate teams. They conclude with a practical conclusion for multinationals: "The findings reinforce the need to view language design as a vital strategic, as well as operational, tool for multinational companies." One possibility could be that MNC's should look at the GII of the subsidiary host country/ region/county to better understand the gender roles in the host society. As I mentioned earlier, the GII has major advantages over survey-based dimensions in capturing local gender roles. Understanding the local gender roles of host societies is an important step in adjusting policies and practices to the local environment. This could also prevent conflicts and other problems that might surface due to a lack of knowledge of a host society. 
Second, Shoham and Lee (2018) show that higher grammatical gender marking of the dominant language in a country increases the wage gap between genders. The finding also shows that grammatical gender marking has an indirect impact on country general income inequality through the gender wage gap. This emphasizes that understanding grammatical gender marking also has implications for policy makers that are interested in reducing society inequality and/or gender wage gaps.

Third, Drori, Manos, Santacreu-Vasut, Shenkar, and Shoham (2018) demonstrate the importance of grammatical gender marking for the growing field of social enterprises. The paper engages with one of the major goals of microfinance organizations attempting to reduce gender-based financial exclusion. In their conclusion for practice, they claim that "Interventions designed to improve state capacity are likely to be most beneficial in countries with high gender marking, because in those environments reducing the cost of providing microfinance loans by improving market infrastructure is particularly beneficial."

To conclude, grammatical gender marking is an important method that improves upon previous methodological attempts to capture gender roles and should/could be used as a major tool in empirical research of gender related issues in society and organizations like board composition and inequality between genders. Nonetheless, it is a simple tool that can help multinationals and practitioners in understanding gender roles in foreign societies. Understanding the gender roles in host countries could prevent costly mistakes that are related to one of the most controversial parts of culture.

\section{References}

Corbett, G. G. 201 1. Chapters 30-32. In M. S. Dryer \& M. Haspelmath (Eds), World Atlas of Linguistic Structures. Munich: Max Planck Digital Library.

Drori, I., Manos, R., Santacreu-Vasut, E., \& Shoham, A. 2019. How does the global microfinance industry determine its targeting strategy across cultures with differing gender values? Journal of World Business (forthcoming).

Drori, I., Manos, R., Santacreu-Vasut, E., Shenkar, O., \& Shoham, A. 2018. Language and market inclusivity for women entrepreneurship: the case of microfinance. Journal of Business Venturing, 33(4): 395-415.

Gay, V., Santacreu-Vasut, E., \& Shoham, A. 2013. The grammatical origins of gender roles. Berkeley Economic History Economic Laboratory Working Paper Series WP2013-03.

Hicks, D. L., Santacreu-Vasut, E., \& Shoham, A. 2015. Does mother tongue make for women's work? Linguistics, household labor, and gender identity. Journal of Economic Behavior \& Organization, 110: 19-44

Hofstede, G. 1980. Culture's Consequences: International Differences in Work-Related Values. Thousand Oaks, CA: Sage.
Santacreu-Vasut, E., Shenkar, O., \& Shoham, A. 2014. Linguistic gender marking and its international business ramifications. Journal of International Business Studies, 45(9): 1170-1178.

Shoham, A., \& Lee, S. M. 2018. The causal impact of grammatical gender marking on gender wage inequality and country income inequality. Business \& Society, 57(6): 1216-1251.

Siewierska, A. 2011 . Gender distinctions in independent personal pronouns. In M. S. Dryer \& M. Haspelmath (Eds), The World Atlas of Language Structures Online. Munich: Max Planck Digital Library.

Wichmann, S., \& Holman, E. W. 2009. Assessing temporal stability for linguistic typological features. Munich: LINCOM Europa.

Dr. Amir Shoham (amir.shoham@temple.edu) is an Associate Professor of Finance and International Business at the Fox School of Business Temple University. Dr. Shoham received his Ph.D. from Ben-Gurion University, Israel. In recent years Dr. Shoham's main research deals with the impact of linguistic gender marking on economic, business, and society outcomes. His linguistic/gender research has appeared in high ranked journals, including the Journal of International Business Studies, Journal of Business Venturing, Journal of Organizational Behavior, among others. 